\title{
Solution of Scalar Riccati Differential Equation of Fractional Order
}

\author{
Fadhel Subhi Fadhel* and Sabreen Hashim Jasim \\ Department of Mathematics and Computer Applications, College of Science, \\ Al-Nahrain University, Baghdad-Iraq. \\ * Corresponding Author: fsf@sc.nahrainuniv.edu.iq.
}

\begin{abstract}
The main objective of this paper is to generalize the scalar Riccati differential equation for factional order derivatives using Caputo definition, and then to find its approximate solution using the variational iteration method. The present work consists of the statement and the proof of the variational iteration formula and then prove of its convergent to the exact solution. Some illustrative examples are considered and simulated using Mathcad 15 computer program.
\end{abstract}

[DOI: 10.22401/ANJS.22.4.09]

Keywords: Scalar Riccati differential equation, variational iteration method, Fractional calculus.

\section{Introduction}

The study of scalar or matrix algebraic and/or differential equations has a great importance, which dates backs from the early days of mathematical analysis, since such equations represent among the most common types of nonlinear equations, especially in mathematical physics and control theory. Also, in recent years, there is a common occurrence of Riccati equations (REs) in variational theory and applied areas of optimal control theory, invariant embedding and dynamic programming [2].

A typical algebraic Riccati equation (ARE) is similar to one of the following types [3], [4]. The generalized Riccati differential equation (RDE) is named after Connt Jacopo Francesco Riccati (1676-1754), who was an Italian nobleman. The one-dimensional Riccati or scalar or generalized RDE has the form

$\dot{X}(t)+q(t) X(t)+r(t) X^{2}(t)-p(t)=0$, for all $t \geq 0$ with initial condition $X(0)=X_{0}$, where $q, r$ and $p$ are scalar functions, [2].

The solution of the matrix Riccati differential equation (MRDE) with initial condition concerned by [5], where this solution is given by using the solution of the algebraic form of the Riccati equation.

Numerical examples are discussed to demonstrate the reliability and ability for the proposed method.

The solution of RDEs by Adomain decomposition method (ADM) to find the analytic solution have been studied by [6]. The solution obtained is the form of a series with simply computable components. Several numerical examples are discussed to find the approximate solution of the RDE and then comparisons were made with Rung - Kutta method and Eulers method. A numerical result shows that the method is quite efficient, more accurate, easily and is practically well suited.

A new application of the variational iteration method (VIM) for one- dimensional quadratic RDE by using Adomian's polynomials in which comparisons with the $\mathrm{ADM}$, and the exact solution have been made by [9].

Applied the VIM to solve general one dimensional RDEs by considering two equations. The first one with a single variable coefficient and the other as a special matrix form and constructed a correction function using VIM by a general Lagrange multiplier. Which can be identified via a variational theory and show that the VIM yields an approximate solution in the form of a quickly convergent series. Furthermore, they have made a comparison RDE with the exact solution and the fourth-order Runge-Kutta method by [8]. Numerical results show that the VIM is very powerful, efficient and more accurate in finding the approximate analytical solution, as well as, numerical solutions for wide classes of linear and nonlinear differential equations.

In this paper, we will prove the existence and uniqness of solution of Riccati differential equations of fractional order. Then solve such equations using Variation iteration method. As well as, the prove of the convergence of the 
sequence of approximate solutions to the exact solution.

\section{Preliminiers}

In this section, some of the most important and basic primitive concepts related to this work are given:

\section{Definition (1), [7]:}

Let $y:[a, b] \rightarrow \mathbb{R}$ be a function, $\alpha$ is a positive real number, $n$ is the integer satisfying, $m-1<\alpha \leq m$, and $\Gamma$ is the Euler gamma function. Then, the left and right Riemann-Liouville fractional integrals of order $\alpha$ are defined respectively by:

The lift Riemann-Liouville fractional integrals of order $\alpha$

$$
{ }_{a} I_{t}^{\alpha} y(t)=\frac{1}{\Gamma(\alpha)} \int_{a}^{t}(t-s)^{\alpha-1} y(s) d s
$$

The right Riemann-Liouville fractional integrals of order $\alpha$

$$
{ }_{b} I_{t}^{\alpha} y(t)=\frac{1}{\Gamma(\alpha)} \int_{t}^{b}(t-s)^{\alpha-1} y(s) d s
$$

\section{Definition (2), [1]:}

he Caputo fractional order derivative of a suitable function $y(t)$ is defined as:

$$
{ }^{c} D_{t}^{\alpha} y(t)=\frac{1}{\Gamma(m-\alpha)} \int_{0}^{t}(t-s)^{m-\alpha-1} y^{(m)}(s) d s
$$

for $a \leq t \leq b$, where $\alpha \in \mathbb{R}^{+}$and $m-1<$ $\alpha \leq m, m=[\alpha]([\alpha]$ is the integer part of $\alpha)$.

It is remarkable here to mention some properties of fractional order derivatives and integrals, which are as follows:

1. $I_{t}^{\alpha} t^{v}=\frac{\Gamma(v+1)}{\Gamma(v+\alpha+1)} t^{\alpha+v}, \alpha \geq 0, m-1<\alpha \leq$ $m, v \geq-1$

2. ${ }^{c} D_{t}^{\alpha}\left(I_{t}^{\beta} y(t)\right)={ }^{c} D_{t}^{\alpha-\beta}(y(t))$.

$\mathrm{y} \in C^{m}[a, b], \alpha, \beta \geq 0, n-1<\alpha \leq n$,

$$
\alpha+\beta \leq m, t>0
$$

3. $\left(I_{t}^{\alpha c} D_{t}^{\alpha} y(t)\right)=y(t)-\sum_{k=0}^{n-1} y^{(k)}(0) \frac{t^{k}}{k !}$ for $m-1<\alpha \leq m, t \in[a, b]$.

\section{Scalar Riccati Differential Equation of Fractional Order}

The Scalar RDE may be generalized and studied with fractional derivatives. That may be called the scalar fractional Riccati differential equation, which has the form:

${ }^{c} D_{t}^{\alpha} X(t)+q(t) X(t)+r(t) X^{2}(t)-p(t)=0$,

$t \geq 0,0<\alpha \leq 1$

with initial condition :

$X(0)=X_{0}$

where $q, r$ and $p$ are given scalar functions.

First, we will study the existence and uniqueness of the solution of the scalar Riccati differential equation (RDE) of fractional order (3) as in the next theorem.

\section{Theorem(1):}

Consider the scalar RDE of fractional order (3), which has the equivalent integral equation

$$
\begin{aligned}
& X(t)=X_{0}+\frac{1}{\Gamma(\alpha)} \int_{t_{0}}^{t}(t-s)^{\alpha-1}[p(s)- \\
& \left.q(s) X(s)-r(s) X^{2}(s)\right] d s
\end{aligned}
$$

and suppose that the integral satisfies Lipschitz condition with respect to $X$ such that $|q(s)|+$ $|r(s)| c]<\frac{\Gamma(\alpha+1)}{\left\|T-t_{0}\right\|^{\alpha}}$, where $\mathrm{c}=\left\|X_{1}\right\|+\left\|X_{2}\right\|$, $\left\|X_{1}{ }^{2}-X_{2}{ }^{2}\right\|=\left\|X_{1}-X_{2}\right\|\left\|X_{1}+X_{2}\right\|$, then there exist a unique solution of equation. (3)

\section{Proof:}

Let $X \in C_{t}^{\alpha}[0, T],{ }^{c} C_{t}^{\alpha}$ is derivative from order $\alpha$ and to prove that:

$$
\begin{gathered}
N X=X_{0}+\frac{1}{\Gamma(\alpha)} \int_{t_{0}}^{t}(t-s)^{\alpha-1}[p(s)- \\
\left.q(s) X(s)-r(s) X^{2}(s)\right] d s
\end{gathered}
$$

is a contraction mapping.

Let $X_{1}, X_{2} \in C_{t}^{\alpha}[0, T]$, then:

$\left\|N X_{1}-N X_{2}\right\|=\| X_{0}+\frac{1}{\Gamma(\alpha)} \int_{t_{0}}^{t}(t-$

$s)^{\alpha-1}\left[p(s)-q(s) X_{1}(s)-r(s) X_{1}^{2}(s)\right] d s-$

$X_{0}-\frac{1}{\Gamma(\alpha)} \int_{t_{0}}^{t}(t-s)^{\alpha-1}\left[p(s)-q(s) X_{2}(s)-\right.$

$\left.r(s) X_{2}^{2}(s)\right] d s \|$

$=\| \frac{1}{\Gamma(\alpha)} \int_{t_{0}}^{t}(t-s)^{\alpha-1}\left[p(s)-q(s) X_{1}(s)-\right.$

$r(s) X_{1}^{2}(s)-p(s)+q(s) X_{2}(s)+$

$\left.r(s) X_{2}{ }^{2}(s)\right] d s \|$

$\leq \frac{1}{\Gamma(\alpha)} \int_{t_{0}}^{t}\left[(t-s)^{\alpha-1}|q(s)|\left\|X_{1}-X_{2}\right\|+\right.$

$\left.|r(s)|\left\|X_{1}{ }^{2}-X_{2}{ }^{2}\right\|\right] d s$ 


$$
\begin{aligned}
& \quad \leq \frac{1}{\Gamma(\alpha)} \int_{t_{0}}^{t}\left[(t-s)^{\alpha-1}|q(s)|\left\|X_{1}-X_{2}\right\|+\right. \\
& \left.|r(s)|\left\|X_{1}-X_{2}\right\|\left\|X_{1}+X_{2}\right\|\right] d s \\
& \leq \frac{1}{\Gamma(\alpha)} \int_{t_{0}}^{t}\left[(t-s)^{\alpha-1}|q(s)|\left\|X_{1}-X_{2}\right\|+\right. \\
& \left.|r(s)|\left\|X_{1}-X_{2}\right\| c\right] d s \\
& \quad=\frac{1}{\Gamma(\alpha)} \int_{t_{0}}^{t}(t-s)^{\alpha-1}[|q(s)|+ \\
& |r(s)| c]\left\|X_{1}-X_{2}\right\| d s \\
& =\frac{1}{\Gamma(\alpha)}[|q(s)|+|r(s)| c] \int_{t_{0}}^{t}(t-s)^{\alpha-1} \\
& \quad\left\|X_{1}-X_{2}\right\| d s \\
& =\frac{1}{\Gamma(\alpha)}[|q(s)|+|r(s)| c] s u p_{s \in[0, T]} \mid X_{1}(s)- \\
& \quad X_{2}(s) \mid \int_{t_{0}}^{t}(t-s)^{\alpha-1} d s \\
& =\frac{1}{\Gamma(\alpha)}[|q(s)|+|r(s)| c] \sup p_{s \in[0, T]} \mid X_{1}(s)- \\
& \quad X_{2}(s) \mid\left(\left.\frac{-(t-s)^{\alpha}}{\alpha}\right|_{t_{0}} ^{t}\right) \\
& \left\|t-t_{0}\right\|^{\alpha}=s u p_{t \in[0, T]}\left|t-t_{0}\right|^{\alpha}, \text { hence: } \\
& \left\|N X_{1}-N X_{2}\right\| \leq \frac{[|q(s)|+|r(s)| c]}{\Gamma(\alpha+1)}\left\|X_{1}-X_{2}\right\|(T- \\
& \left.t_{0}\right)^{\alpha} \\
& \quad=\frac{[|q(s)|+|r(s)| c]\left(T-t_{0}\right)^{\alpha}}{\Gamma(\alpha+1)}\left\|X_{1}-X_{2}\right\|
\end{aligned}
$$

Then $N$ is a contraction if $0<\frac{[|q(s)|+|r(s)| c]\left\|T-t_{0}\right\|^{\alpha}}{\Gamma(\alpha+1)}<1$

i.e., if $[|q(s)|+|r(s)| c]<\frac{\Gamma(\alpha+1)}{\left\|T-t_{0}\right\|^{\alpha}}$

So, using Banach fixed point theorem, $N$ has a unique fixed point $X$, i.e., $N X=X$

Thus, the scalar RDE of fractional order (3) has a unique solution.

\section{Variational Iteration Method for solving Scalar Fractional RDE}

In this section, the VIM will be used to find the approximate solution of equation (3), as it is stated and proved in the next theorem:

\section{Theorem(2):}

Consider the RDE of fractional order (3). The related variational iteration formula is given by:

$$
\begin{aligned}
& X_{n+1}(t)=X_{n}(t)- \\
& I^{\alpha}\left\{{ }^{C} D^{\alpha} X_{n}(t)+q(t) X_{n}(t)+r(t) X_{n}^{2}(t)-\right. \\
& p(t)\}
\end{aligned}
$$

where $X_{n}$ is the $n^{\text {th }}$ approximation and ${ }_{a} I_{t}^{\alpha}$ is the lift Riemann-Liouville 's fractional integral of order $\alpha \in(0,1]$.

\section{Proof:}

Recall that:

$$
\begin{aligned}
& { }^{C} D^{\alpha} X_{n}(t)+q(t) X_{n}(t)+r(t) X^{2}{ }_{n}(t)- \\
& p(t)=
\end{aligned}
$$

Multiply the above equation by the general Lagrange multiplier $\lambda(t, s)$ yields to:

$$
\begin{gathered}
\lambda(t, s)\left\{{ }^{C} D^{\alpha} X_{n}(t)+q(t) X_{n}(t)+r(t)\right. \\
\left.X^{2}{ }_{n}(t)-p(t)\right\}=0 \ldots \ldots \ldots \ldots \ldots \ldots \ldots \ldots \ldots \ldots \ldots \ldots \ldots \ldots \ldots \ldots \ldots \ldots \ldots \ldots \ldots
\end{gathered}
$$

Now, take lift Riemann-Liouville fractional integrals of order $\alpha$ to the both sides of equation (6), which give to:

$$
\begin{aligned}
& \quad{ }_{a} I_{t}^{\alpha}\left[\lambda ( t , s ) \left\{{ }^{C} D^{\alpha} X_{n}(t)+q(t) X_{n}(t)+\right.\right. \\
& \left.\left.r(t) X^{2}{ }_{n}(t)-p(t)\right\}\right]=0
\end{aligned}
$$

Then, the correction functional for equation (3) will be read as follows:

$$
\begin{aligned}
& X_{n+1}(t)=X_{n}(t)+{ }_{a} I_{t}^{\alpha} \lambda(t, s)\left\{{ }^{C} D^{\alpha} X_{n}(t)+q(t)\right. \\
& \left.X_{n}(t)+r(t) \tilde{X}_{n}^{2}(t)-p(t)\right\}=0 \ldots \ldots \ldots \ldots . .(7)
\end{aligned}
$$

In this case the value of $\lambda$ cannot be evaluated easily form equation (7), which will give a functional with fractional integral. Therefore, the approximation of the correctional functional may be expressed as follows:

$$
\begin{aligned}
& X_{n+1}(t)=X_{n}(t)+\int_{0}^{t} \lambda(t, s)\left\{\frac{d X_{n}(s)}{d s}+\right. \\
& \left.q(s) X_{n}(s)+r(s) \tilde{X}_{n}^{2}(s)-p(s)\right\} d s
\end{aligned}
$$

for all $n=0,1,2, \ldots$; where $\lambda$ is the general Lagrange multiplier for the initial value problem:

$$
\begin{aligned}
& \dot{X}(t)+F(t, X(t))=0, \text { where } \\
& F(t, X(t))=q(t) X+r(t) \tilde{X}^{2}-p(t)
\end{aligned}
$$

Which will give by $\lambda(t, s)=-1$ and

$$
\begin{aligned}
X_{n+1}(t) & =X_{n}(t)-I^{\alpha}\left\{{ }^{C} D^{\alpha} X_{n}(t)+\right. \\
& \left.p(t) X_{n}(t)+q(t) X_{n}^{2}(t)-c(t)\right\}
\end{aligned}
$$

\section{Theorem (3):}

Let $X, X_{n} \in\left(C^{\alpha}[0, T],\|.\|_{\infty}\right), n=0,1 \ldots$ be respectively the exact and approximate solutions of the scalar RDE of fractional order (3). If $E_{n}(t)=X_{n}(t)-X(t)$, and the nonlinear operator $N X=q X^{2}$ satisfies condition, such 
that $\quad\left[\frac{(\|q(t)\|+c\|r(t)\|)}{\Gamma(\alpha)}\right]^{n} T^{n \alpha}<1, \quad$ where $c=\left\|X_{n}\right\|+\|X\|$ then the sequence of approximate solutions $\left\{X_{n}\right\}, n=0,1, \ldots$; converge to exact solution $X$.

\section{Proof :}

Consider the RDE of fractional order: ${ }^{C} D^{\alpha} X(t)+q(t) X(t)+r(t) X^{2}(t)=p(t)$, $X(0)=X_{0}$

Where the approximate solutions using the VIM is given by:

$$
\begin{gathered}
X_{n+1}(t)=X_{n}(t)-I^{\alpha}\left\{{ }^{C} D^{\alpha} X_{n}(t)+\right. \\
\left.q(t)(t))+r(t) X_{n}^{2}(t)-p(t)\right\}, \\
0<\alpha \leq 1
\end{gathered}
$$

Since $X$ is the exact solution of the integral equations of fractional order, hence:

$$
\begin{gathered}
X(t)=X(t)-I^{\alpha}\left\{{ }^{C} D^{\alpha} X(t)+q(t) X(t)+\right. \\
\left.r(t) X^{2}(t)-p(t)\right\}
\end{gathered}
$$

Hence, subtracting $X$ from $X_{n+1}$ yields to:

$$
\begin{gathered}
X_{n+1}(\mathrm{t})-\mathrm{X}(\mathrm{t})=\mathrm{X}_{n}(\mathrm{t})-\mathrm{X}(\mathrm{t})- \\
\mathrm{I}^{\alpha}\left\{{ }^{\mathrm{C}} \mathrm{D}^{\alpha} \mathrm{X}_{\mathrm{n}}(\mathrm{t})-{ }^{\mathrm{C}} \mathrm{D}^{\alpha} \mathrm{X}(\mathrm{t})+\right. \\
q(\mathrm{t}) \mathrm{X}_{\mathrm{n}}(\mathrm{t})-\mathrm{q}(\mathrm{t}) \mathrm{X}(\mathrm{t})+r(t) \\
\left.\mathrm{X}^{2}{ }_{\mathrm{n}}(\mathrm{t})-\mathrm{r}(\mathrm{t}) \mathrm{X}^{2}(\mathrm{t})+\mathrm{p}(\mathrm{t})-\mathrm{p}(\mathrm{t})\right\}
\end{gathered}
$$

Hence:

$E_{n+1}(t)=E_{n}(t)-I^{\alpha}\left\{{ }^{C} D^{\alpha} E_{n}(t)+\right.$

$\left.q(t) E_{n}(t)+r(t)\left(X_{n}^{2}-X^{2}\right)\right\}$

$=E_{n}(0)-I^{\alpha}\left\{q(t) E_{n}(t)+r(t)\left(X_{n}+\right.\right.$

$\left.X)\left(X_{n}-X\right)\right\}$

$=E_{n}(t)-I^{\alpha}\left\{q(t) E_{n}(t)+r(t)\left(X_{n}+X\right) E_{n}\right\}$

$E_{n}(0)=X_{n}(0)-X(0)$, with $X_{n}(0)=X(0)$, then $E_{n}(0)=0$.

Therefore:

$$
\begin{aligned}
E_{n+1}(t)= & -I^{\alpha}\left\{q(t) E_{n}(t)\right\}-I^{\alpha}\left\{r ( t ) \left(X_{n}+\right.\right. \\
& \left.X) E_{n}(t)\right\} \\
= & -\frac{1}{\Gamma(\alpha)} \int_{0}^{t}(t-s)^{\alpha-1} q(s) E_{n}(s) d s- \\
& \frac{1}{\Gamma(\alpha)} \int_{0}^{t}(t-s)^{\alpha-1} r(s)\left(X_{n}+\right. \\
& X) E_{n}(s) d s
\end{aligned}
$$

Hence, upon taking the supremum norm, we have:

$$
\left\|E_{n+1}(t)\right\|_{\infty} \leq \frac{1}{\Gamma(\alpha)} \int_{0}^{t}(t-s)^{\alpha-1}\left\|E_{n}(s)\right\|_{\infty}
$$$$
\|q(s)\| d s+\frac{1}{\Gamma(\alpha)} \int_{0}^{t}\|t-s\|^{\alpha-1} \|\left(X_{n}+\right.
$$

$X)\|\| E_{n}(s)\left\|_{\infty} \quad\right\| r(s) \| d s$

$$
\begin{aligned}
& \leq \frac{\|q(t)\|}{\Gamma(\alpha)} \int_{0}^{t}(t-s)^{\alpha-1}\left\|E_{n}(s)\right\|_{\infty} d s \\
& +\frac{1}{\Gamma(\alpha)} \int_{0}^{t} \sup _{s \in[0, T]}|t-s|^{\alpha-1} \\
& \left(\left\|X_{n}\right\|+\|X\|\right)\left\|E_{n}(s)\right\|_{\infty}\|r(t)\| d s
\end{aligned}
$$

$\leq \frac{\|q(t)\|}{\Gamma(\alpha)} \int_{0}^{t}\|t-s\|^{\alpha-1}\left\|E_{n}(s)\right\|_{\infty} d s+$

$\frac{1}{\Gamma(\alpha)} \int_{0}^{t} \sup _{s \in[0, T]}|t-s|^{\alpha-1} c$

$\left\|E_{n}(s)\right\|_{\infty}\|r(t)\| d s$

$\leq(\|q(t)\|+c\|r(t)\|) \frac{1}{\Gamma(\alpha)}$

$\int_{0}^{t} \sup _{s \in[0, T]}|t-s|^{\alpha-1}\left\|E_{n}(s)\right\|_{\infty} d s$

$\leq \frac{(\|q(t)\|+c\|r(t)\|)}{\Gamma(\alpha)} \int_{0}^{t} t^{\alpha-1}\left\|E_{n}(s)\right\|_{\infty} d s$

$\leq$

$\frac{(\|q(t)\|+c\|r(t)\|)}{\Gamma(\alpha)} t^{\alpha-1} \int_{0}^{t}\left\|E_{n}(s)\right\|_{\infty} d s$

Where $n=0,1,2, \ldots$;

Now, if $n=0$, then:

$\left\|E_{1}(t)\right\|_{\infty} \leq \frac{(\|q(t)\|+c\|r(t)\|)}{\Gamma(\alpha)} t^{\alpha-1}$

$\int_{0}^{t}\left\|E_{0}(s)\right\|_{\infty} d s$

$\leq \frac{(\|q(t)\|+c\|r(t)\|)}{\Gamma(\alpha)} t^{\alpha-1}$

$\int_{0}^{t} \sup _{s \in[0, T]}\left|E_{0}(s)\right|_{\infty} d s$

$\leq \frac{(\|q(t)\|+c\|r(t)\|)}{\Gamma(\alpha)}$

$t^{\alpha-1} \sup _{s \in[0, T]}\left|E_{0}(s)\right| \int_{0}^{t} d s$

$\leq \frac{(\|q(t)\|+c\|r(t)\|)}{\Gamma(\alpha)}$

$t^{\alpha} \sup _{s \in[0, T]}\left|E_{0}(s)\right|$

Also, for $n=1$, we have:

$\left\|E_{2}(t)\right\|_{\infty} \leq \frac{(\|q(t)\|+c\|r(t)\|)}{\Gamma(\alpha)} t^{\alpha-1}$

$\int_{0}^{t}\left\|E_{1}(s)\right\|_{\infty} d s$

$\leq \frac{(\|q(t)\|+c\|r(t)\|)}{\Gamma(\alpha)} t^{\alpha-1}$

$\int_{0}^{t} \frac{(\|q(t)\|+c\|r(t)\|)}{\Gamma(\alpha)} s^{\alpha}$

$\sup _{s \in[0, T]}\left|E_{0}(s)\right|_{\infty} d s$

$\leq\left[\frac{(\|q(t)\|+c\|r(t)\|)}{\Gamma(\alpha)}\right]^{2}$

$t^{\alpha-1} \sup _{s \in[0, T]}\left|E_{0}(s)\right| \int_{0}^{t} s^{\alpha} d s$

$\leq\left[\frac{(\|q(t)\|+c\|r(t)\|)}{\Gamma(\alpha)}\right]^{2} \frac{t^{2 \alpha}}{\alpha+1}$

$\sup _{s \in[0, T]}\left|E_{0}(s)\right|$

Similarly, for $n=2$, then: 


$$
\begin{aligned}
&\left\|E_{3}(t)\right\|_{\infty} \leq \frac{(\|q(t)\|+c\|r(t)\|)}{\Gamma(\alpha)} t^{\alpha-1} \\
& \int_{0}^{t}\left\|E_{2}(s)\right\|_{\infty} d s \\
& \leq \frac{(\|q(t)\|+c\|r(t)\|)}{\Gamma(\alpha)} t^{\alpha-1} \\
& \int_{0}^{t}\left[\frac{(\|q(t)\|+c\|r(t)\|)}{\Gamma(\alpha)}\right]^{2} \\
& \frac{s^{2 \alpha}}{\alpha+1} s u p_{s \in[0, T]}\left|E_{0}(s)\right|_{\infty} d s \\
& \leq {\left[\frac{(\|q(t)\|+c\|r(t)\|)}{\Gamma(\alpha)}\right]^{3} t^{\alpha-1} } \\
& \sup _{s \in[0, T]} \mid E_{0}(s) \mid \int_{0}^{t} \frac{s^{2 \alpha}}{\alpha+1} d s \\
& \leq {\left[\frac{(\|q(t)\|+c\|r(t)\|)}{\Gamma(\alpha)}\right]^{3} t^{\alpha-1} } \\
& \sup _{s \in[0, T]} \mid E_{0}(s) \mid \frac{t^{2 \alpha+1}}{3 \alpha+1} \\
& \leq {\left[\frac{(\|q(t)\|+c\|r(t)\|)}{\Gamma(\alpha)}\right]^{3} } \\
& \frac{t^{3 \alpha}}{3 \alpha+1} s u p_{s \in[0, T]}\left|E_{0}(s)\right|
\end{aligned}
$$

and so on, in general, for any natural number $n$

$$
\begin{aligned}
\left\|E_{n}(t)\right\| \leq & {\left[\frac{(\|q(t)\|+c\|r(t)\|)}{\Gamma(\alpha)}\right]^{n} } \\
& \frac{t^{(n+1) \alpha}}{((2 n-1) \alpha+1)} \\
\sup _{s \in[0, T]}\left|E_{0}(s)\right| & {\left[\frac{(\|q(t)\|+c\|r(t)\|)}{\Gamma(\alpha)}\right]^{n} } \\
\leq & \frac{T^{(n+1) \alpha}}{((2 n-1) \alpha+1)} \sup _{s \in[0, T]}\left|E_{0}(s)\right|
\end{aligned}
$$

If $\|q(t)\|+c\|r(t)\| T^{\alpha}<1$, and as $n \rightarrow \infty$, then $\left[\frac{(\|q(t)\|+c\|r(t)\|)}{\Gamma(\alpha)}\right]^{n} T^{(n+1) \alpha} \rightarrow 0, \quad$ which implies that $\left\|E_{n}(t)\right\|_{\infty} \rightarrow 0$ i.e., $X_{n}(t) \rightarrow X(t)$, as $n \rightarrow \infty$, which means that the sequence of approximate solution using the equation (3) converge to the exact solution

\section{Illustrative Examples}

In this section, some illustrative examples will be considered and simulated using computer programs written in Mathcad15.

\section{Example (1):}

Consider the scalar RDE [10]:

${ }^{c} D_{t}^{\alpha} X(t)=1+t^{2}-X^{2}(t), \in \alpha[0,1], \mathrm{t} \in[0,1]$

With initial condition: $X_{0}(\mathrm{t})=1$

Compare between equation (9) and equation (3) yields:

$q(t)=0, r(t)=1, \mathrm{p}(t)=1+t^{2}$
For the comparison purpose, the exact solution of equation (3):

$X(t)=t+\frac{e^{-t^{2}}}{1+\int_{0}^{t} e^{-u^{2}} d u}$

In the VIM, we have freely to choose the initial approximate solution and hence starting with the initial solution $X_{0}(\mathrm{t})=1$ and by applying the VIM for equation (9), we get if $\alpha=0.8$, then the first approximate solution.

$X_{1}(t)=X_{0}(t)-I^{0.8}\left\{{ }^{C} D^{0.8} X_{0}(s)+X_{0}^{2}(s)-\right.$ $\left.s^{2}-1\right\}=1.07 \cdot t^{0.8}+0.426 \cdot t^{2.8}$

and also the second approximate solution

$X_{2}(t)=X_{1}(t)-I^{0.8}\left\{{ }^{C} D^{0.8} X_{1}(s)+X_{1}^{2}(s)-\right.$ $\left.s^{2}-1\right\}$

$=-0.275 \cdot t^{4.4}-0.553 \cdot t^{2.4}+1.074$

$t^{0.8}+0.335 \cdot t^{2.8}-0.04 \cdot t^{6.4}+0.426 \cdot t^{2.8}$

and so on we may compute $X_{3}, X_{4}, \ldots$ In Table (1) we present the $4^{\text {th }}$ approximate solution.

Table (1)

Comparison between the exact and approximate solution for $X_{4}(t)$.

\begin{tabular}{||c||c|c|}
\hline $\mathbf{t}$ & Exact solution & $\boldsymbol{X}_{\mathbf{4}}(\boldsymbol{t})$ \\
\hline \hline 0 & 0 & 0 \\
\hline 0.1 & 1.000317 & 0.165 \\
\hline 0.2 & 1.0024198 & 0.279 \\
\hline 0.3 & 1.0077945 & 0.375 \\
\hline 0.4 & 1.0176508 & 0.462 \\
\hline 0.5 & 1.0329575 & 0.543 \\
\hline 0.6 & 1.0544668 & 0.619 \\
\hline 0.7 & 1.0827274 & 0.692 \\
\hline 0.8 & 1.1180925 & 0.764 \\
\hline 0.9 & 1.1607239 & 0.837 \\
\hline 1 & 1.204 & 0.918 \\
\hline
\end{tabular}

Also, sketch of the approximate solutions are presented in Fig. (1) 


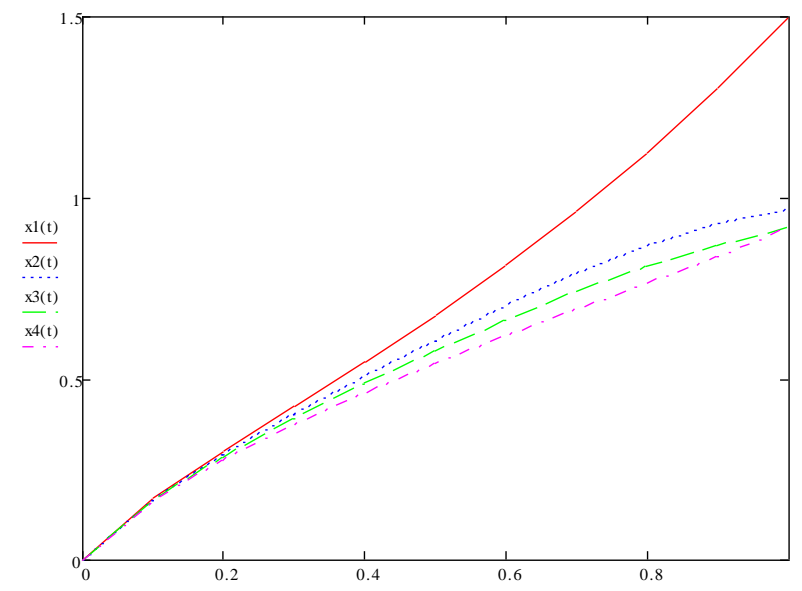

Fig.(1): The approximate solutions of example (1) by using the proposed method at different values of $\alpha$ at $\alpha=0.8$.

\section{Example (2):}

Consider the nonlinear RDE : ${ }^{c} D_{t}^{\alpha} X(t)+t X(t) \quad+X^{2}(t)=t^{2}, \quad 0<\alpha \leq 1$, $t \in[0,1]$

with initial condition $X_{0}(t)=1$

Comparing equation (10) with equation (3), we get $p(t)=t, q(t)=1, c(t)=t^{2}$. Then the VIM for equation (10) is:

$$
\begin{gathered}
X_{n+1}=X_{n}-I^{\alpha}\left\{{ }^{C} D^{\alpha} X_{n}(s)+s X_{n}(s)+\right. \\
\left.X_{n}{ }^{2}(s)-s^{2}\right\}
\end{gathered}
$$

For $\alpha=0.2$,then the approximates solution, are given by

$$
\begin{aligned}
X_{1}= & X_{0}-I^{0.2}\left\{{ }^{C} D^{0.2} X_{0}(s)+s X_{0}(s)+\right. \\
& \left.X_{0}^{2}(s)-s^{2}\right\} \\
= & 0.825 \cdot t^{2.2} \\
X_{2}= & X_{1}-I^{0.2}\left\{{ }^{C} D^{0.2} X_{1}(s)+s X_{1}(s)+\right. \\
& \left.X_{1}^{2}(s)-s^{2}\right\} \\
= & -0.257 \cdot t^{5.2}+1.65 \cdot t^{2.2}-0.248 \\
& t^{2.2}-0.454 \cdot t^{3.7}:
\end{aligned}
$$

and so on we may compute $X_{3}, X_{4}, \ldots$ which will be more complicated to be evaluated and so we present then numerical results for the $4^{\text {th }}$ approximate solution, as it is presented in Table (2)
Table (2)

Comparison between the exact and approximate solution for $\mathrm{X}_{4}(t)$.

\begin{tabular}{|c||c|c|}
\hline $\boldsymbol{t}$ & Exact solution & $\boldsymbol{X}_{\mathbf{4}}(\boldsymbol{t})$ \\
\hline \hline 0 & 0 & 0 \\
\hline 0.1 & $3.32666037 \cdot 10^{-4}$ & 0.013 \\
\hline \hline 0.2 & $2.64525494 \cdot 10^{-3}$ & 0.056 \\
\hline 0.3 & $8.83672133 \cdot 10^{-3}$ & 0.124 \\
\hline 0.4 & 0.02064171 & 0.206 \\
\hline 0.5 & 0.03954439 & 0.286 \\
\hline \hline 0.6 & 0.06669229 & 0.346 \\
\hline \hline 0.7 & 0.10281696 & 0.372 \\
\hline 0.8 & 0.14817032 & 0.358 \\
\hline \hline 0.9 & 0.20248288 & 0.312 \\
\hline \hline 1 & 0.26493764 & 0.251 \\
\hline
\end{tabular}

Also, sketch of the approximate solutions are presented in Fig. (2)

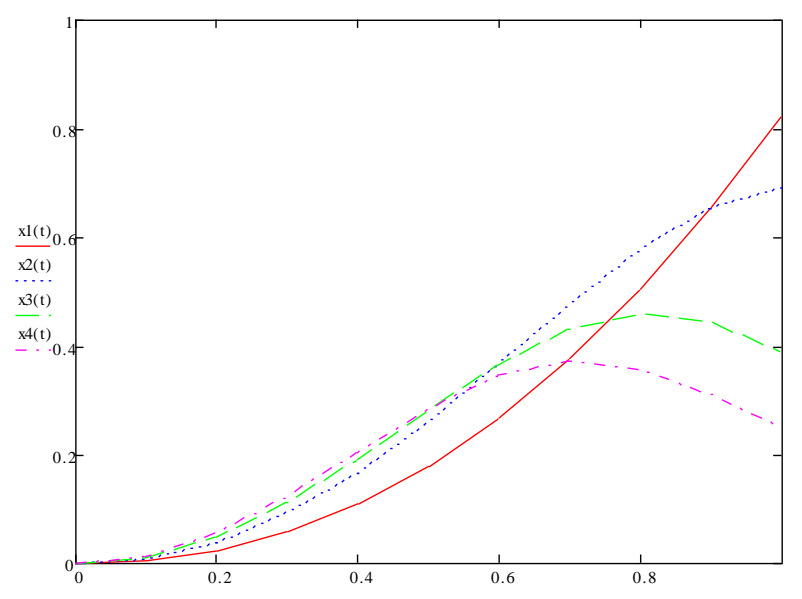

Fig.(2): The approximate solution of example (2) by using the proposed method at different values of $\alpha$ at $\alpha=0.2$.

If $\alpha=1$, then the solution given in Table (3): $X_{1}=X_{0}-$

$$
\begin{aligned}
& I^{1}\left\{{ }^{C} D^{1} X_{0}(s)+s X_{0}(s)+X_{0}{ }^{2}(s)-s^{2}\right\} \\
& =\frac{t^{3}}{3} \\
& X_{2}=X_{1}- \\
& I^{1}\left\{{ }^{C} D^{1} X_{1}(s)+s X_{1}(s)+X_{1}^{2}(s)-s^{2}\right\} \\
& =\frac{t^{3}}{3}-\frac{t^{5}}{15}-\frac{t^{7}}{63}
\end{aligned}
$$

and so on we may compute $X_{3}, X_{4}, \ldots$ which will be more complicated and the results for $\mathrm{X}_{4}(\mathrm{t})$ are presented in Table (3). 
Table (3)

Comparison between the exact and approximate solution for ten iteration.

\begin{tabular}{|c|c|c||}
\hline $\boldsymbol{t}$ & Exact solution & $\boldsymbol{X}_{\mathbf{4}}(\boldsymbol{t})$ \\
\hline \hline 0 & 0 & 0 \\
\hline 0.1 & $3.32666037 \cdot 10^{-4}$ & $3.327 \cdot 10^{-4}$ \\
\hline 0.2 & $2.64525494 \cdot 10^{-3}$ & $2.645 \cdot 10^{-3}$ \\
\hline 0.3 & $8.83672133 \cdot 10^{-3}$ & $8.837 \cdot 10^{-3}$ \\
\hline 0.4 & 0.02064171 & 0.021 \\
\hline 0.5 & 0.03954439 & 0.04 \\
\hline 0.6 & 0.06669229 & 0.067 \\
\hline 0.7 & 0.10281696 & 0.103 \\
\hline 0.8 & 0.14817032 & 0.148 \\
\hline 0.9 & 0.20248288 & 0.202 \\
\hline 1 & 0.26493764 & 0.265 \\
\hline
\end{tabular}

Also, sketch of the approximate solutions are presented in Fig. (3)

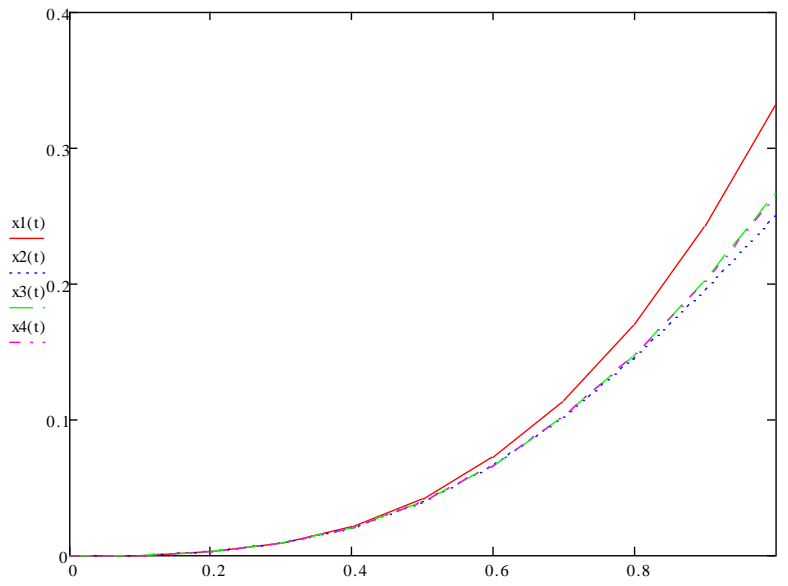

Fig.(3): The approximate solution of example (2) by using the proposed method at different values of $\alpha$ at $\alpha=1$.

\section{Conclusion}

The existence and uniqueness theorem of the solution of scalar RDE and fractional order RDE are proved in this paper two and then find its approximate by applying the VIM by deriving the general form of the iterative approximate sequence of solutions for this equation and then proved the convergence of the obtained sequence of approximate solutions to the exact solution. This proof is based on using the mathematical induction to derive a general formula. Numerical examples have been considered to explain how to apply this method for solving scalar RDE of fractional order and reliable results are obtained. For scalar RDE, we implemented the VIM to find approximate analytical solution and comparative solution with the exact solution, if exist, have made.

\section{References}

[1] Caputo M., "Linear models of dissipation whose $\mathrm{Q}$ is almost frequency independent II", Geophys. J. Roy. Astronom. Soc. 13, p. 529-539, 1967.

[2] Reid, W. T., "Riccati differential equations". Mathematics in science and engineering, 86. New York, Academic Press, 1972.

[3] Lancaster, P. and Rodman L., "Solution of the continuous and discrete time algebraic Riccati equation", a review, In the Riccati equation, edited: Sergio B., Alan L. and Lan C. W., Springer-Verlang, 1991.

[4] Rodman, L., "Algebraic Riccati equations", Claredon Press, Oxford, 1995.

[5] Nazazadeh, J., Razzaghi, M., and Nikravesh, K., "solution of the matrix Riccati equation for the linear quadratic control problems". Math1. Comput. Modelling, 27(7), 51-55, 1998.

[6] Bulut, H. and Evans, D., "On the solution of Riccati equation by the decomposition method", International Journal of computer Mathematics, 79(1), 103-109, 2002.

[7] Loverro, A., Fractional Calculus: History, Definitions and Applications for the Engineer, Department of Aerospace and Mechanical Engineering, University of Notre Dame, Notre Dame, IN 46556, USA, May 8, 2004.

[8] Batiha, B., Noorani, M. and Hashim, I., "Application of variational iteration method to a general Riccati equation", International Mathematical Forum, 2(56), 5279-2770, 2007.

[9] Abbasbandy, S., "Anew application of He's variational iteration method for quadratic Riccati differential equation by using Adomian's polynomials", Journal of Computational and Applied Mathematics, 207(1), 59-63, 2007.

[10] Geng, F., Lin, y. and Gui, M.,. A piecewise variational iteration method for Riccati differential equations, computers and Mathematics with Applications, 58(1112), 2518-2522, (2009). 